\title{
Chrome RLZ and Unknown Features
}

\author{
A. Sankara Narayanan \\ Department of Information Technology \\ Salalah College of Technology, Salalah, Sultanate of Oman
}

\begin{abstract}
The web browser is arguably the most important piece of software on your computer. Web browsers originated as applications that people used to view static web sites sequentially. All are spend much of your time online inside a browser: when you search, chat, email, shop, bank, read the news, and watch videos online, and do all this using a browser. This paper formulates content chrome RLZ tracking and hidden features. The paper studies RLZ tracking systematically by delivering advertisements and RLZ to identify the user languages. Chrome is also immensely easy to use; it tries to be nothing more than just a browser. This paper introduces hidden commands and address bar simple arithmetic calculations.
\end{abstract}

\section{Keywords}

Google Chrome, RLZ, Chrome Features

\section{INTRODUCTION}

A web browser is a software application that enables users to access the World Wide Web (WWW). Google Chrome is a freeware web browser developed by Google that uses the WebKit layout engine. It was first released as a beta version for Microsoft Windows on September 2, 2008, and the public stable release was on December 11, 2008. As of January 2012, Google Chrome has approximately $25-28 \%$ worldwide usage share of web browsers, making it the second or the third most widely used browser [13]. Google also expressed hope that other browsers would adopt the V8 JavaScript engine to improve web application performance. The Google authored portion of Chromium is released under the permissive Berkeley Software Distribution (BSD - licenses are family of permissive free software licenses) license [14], other portions of the source code are subject to a variety of open source licenses. Chromium implements a similar feature set as Chrome, but lacks built-in automatic updates, built-in PDF reader and Google branding, as well as built-in Google tracking [15].

Chrome was assembled from 25 different code libraries from Google and third parties such as Mozilla's Netscape Portable Runtime, Network Security Services, NPAPI, as well as SQLite and a number of other open-source projects [16]. Chrome uses the WebKit rendering engine to display web pages. Chrome is tested internally with unit testing, automated user interface testing of scripted user actions, fuzz testing, as well as WebKit's layout tests. Chrome is an open source browser project that aims to build a safer, faster, and more stable way for all Internet users to experience the web. As for the internet users not updating their Chrome and let the chance of virus attacks increases, Google came out using one of the open source systems which is called the Omaha. This software can automatically check the user's software for every five hours, and it can dramatically decrease the chance of users affected by the attackers [18]. Google's Chrome Web browser though just keeps getting better with every new release.
The BSI (Germany's federal office of information security, known by the German initials of BSI) has announced that it recommends Google's Chrome browser for German citizens as the most secure for surfing the web [17]. We can see the developments of Google Chrome throughout the years and its popularity is booming. It is also a pleasure to imagine how fantastic experience Chrome will be giving us.

\section{RLZ}

The RLZ information includes a non-unique promotional tag that contains information about how Chrome was obtained, the week when Chrome was installed, and the week when the first search was performed. This parameter does not uniquely identify your computer, nor is it used to target advertising. This information is used to understand the effectiveness of different distribution mechanisms, such as downloads directly from Google vs. other distribution channels. This cannot be disabled so long as your search provider is Google. If your default search provider is not Google, then searches performed using the address bar will go to your default search provider, and will not include this RLZ parameter. The RLZ means "Rules" project is a library for grouping promotion event signals and anonymous user cohorts. The tag looks similar to "1T4AAAA enUS236US239". This non-unique tag is included when performing searches via Google (the tag appears as a parameter beginning with "rlz=" when triggered from the Omnibox, or as an "x-rlz-string" HTTP header). We use this information to help us measure the searches and Chrome usage driven by a particular promotion [9].

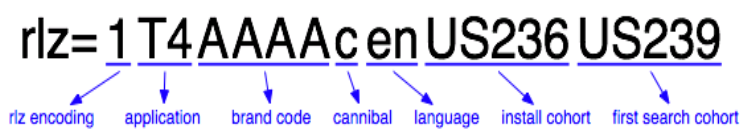

\section{Fig 1: RLZ string}

Client applications with the RLZ library can use explicit cohort tagging to manage promotion analysis. A client application with a particular tag can transmit that tag as it chooses for payments and analysis purposes. As an example, the RLZ parameter "rlz=1T4AAAA_enUS236" indicates the client application is Toolbar version 4, distributed with AAAA software bundle, English version, to a US user [10].

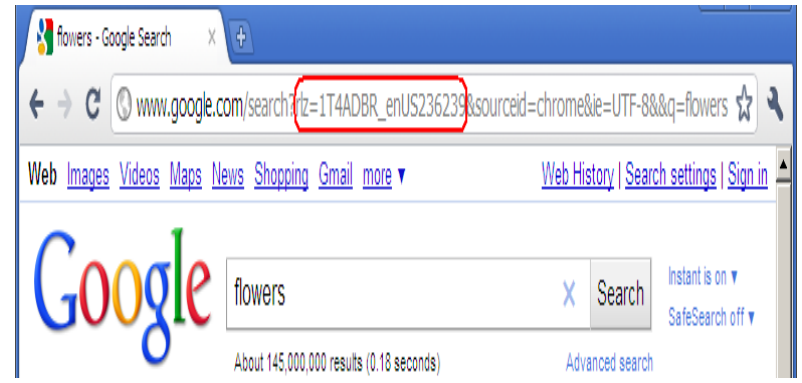

Fig 2: RLZ string with URL address bar 
Installations of Google Chrome obtained via promotional campaigns also send a token when you first launch Chrome and when you first search from Google. The same token will be sent if Chrome is later reinstalled and is only sent at first launch and at first use of the Omnibox after reinstallation or reactivation. Rather than storing the token on the computer, it is generated when necessary by using built in system information that is scrambled in an irreversible manner. Google Chrome uses a software library called "RLZ" to generate and send this information [9].

\section{UNKNOWN FEATURES}

\subsection{Omni box}

The browser has an address bar with auto-completion features called 'omnibox'; Google says it offers search suggestions ("Omni" is a prefix meaning "all", as in "omniscient" - "allknowing"). The simple way to explain separate search box is combined with the address bar in what it calls the "Omnibox." Pure google search simply put a "?" before your query.

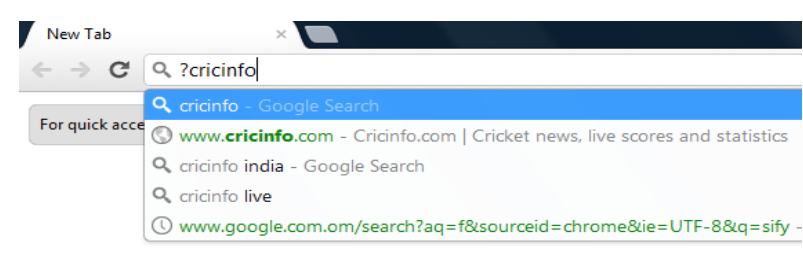

Fig 3: Omnibox simple query

\subsection{Quick calculation}

We can do simple mathematical calculations like addition, subtraction, multiplication, finding percentage and more. Quick calculation results from address bar. It is faster than opening up your calculator. Just type (e.g. $\sin (30)+\cos (30)$ ) the result will come up automatically. Google chrome address bar only applicable these features.

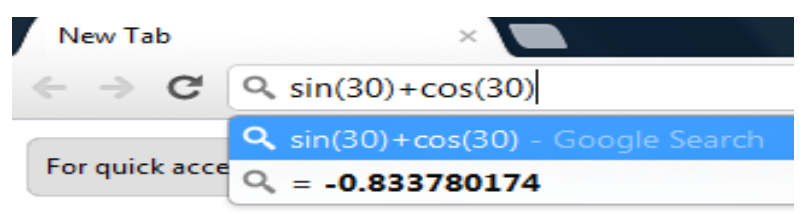

Fig 4: Address bar calculator

\subsection{Drag downloaded files}

Easily drag downloaded files from Chrome to your desktop or any other folder on your computer. That means, from now on, no need to find the folder or change the download location each time.

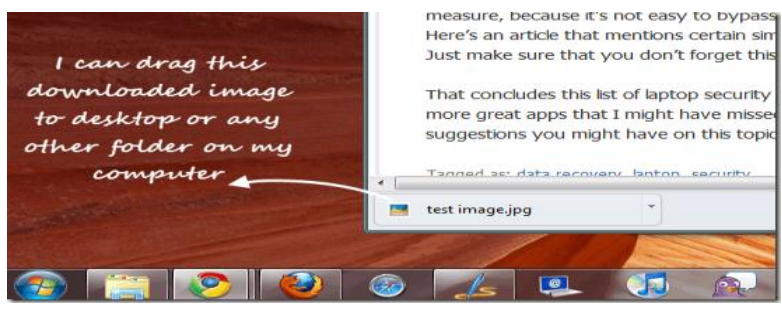

Fig 5: Downloaded image to desktop

\subsection{Resize Multi-line textboxes}

This feature is related to Multi-line Text Boxes. Google has observed this carefully and allowed the user to change the size of such Multiline Text Boxes according to users' requirements. It is very easy to use this feature. A lot of times, the text boxes on webpage's are annoying. They are too small and just typed a few lines; we get a scroll bar which is irritating. On Chrome, actually drag that box from the corner and make it bigger.

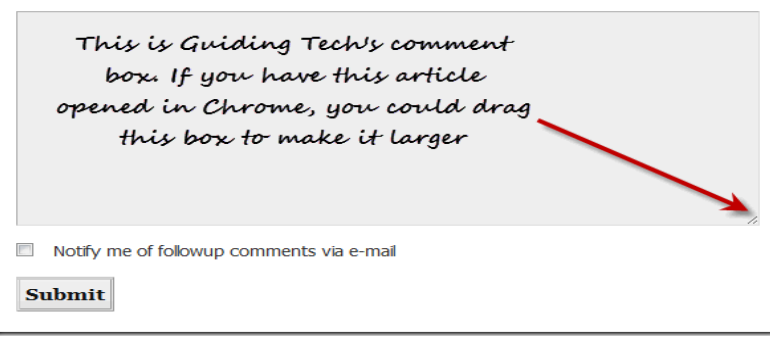

Fig 6: Resize the text box

\subsection{Create application shortcut}

Creating desktop shortcuts for frequently visited sites is a smart move that saves time. Google Chrome provides an easy option to create desktop application shortcuts. It's not the same as creating the shortcut of a webpage in other browsers. These shortcuts help you open the site in a chrome window dedicated to them. Launch any website from the desktop, start menu, quick launch bar. Open any site and choose "Create application shortcut" from the Wrench menu.

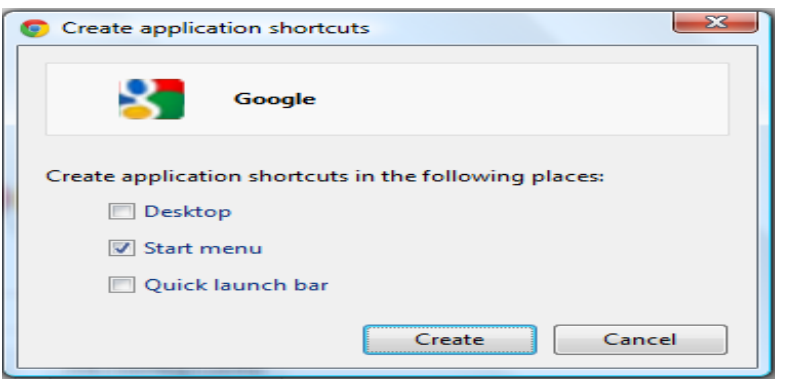

Fig 7: Create standalone application shortcut

\subsection{Developer tools}

The Developer Tools are available in Chrome and Safari, allows web developers and programmers deep access into the internals of the browser and their web application. The Developer Tools are part of the open source Webkit project. This article applies to the Google Chrome browser on windows. To access the developers' tools, open a web page in google chrome. Then select the Wrench menu at the top of the top right of your browser window, then select tools $\rightarrow$ developers tools (Control-Shift-I).

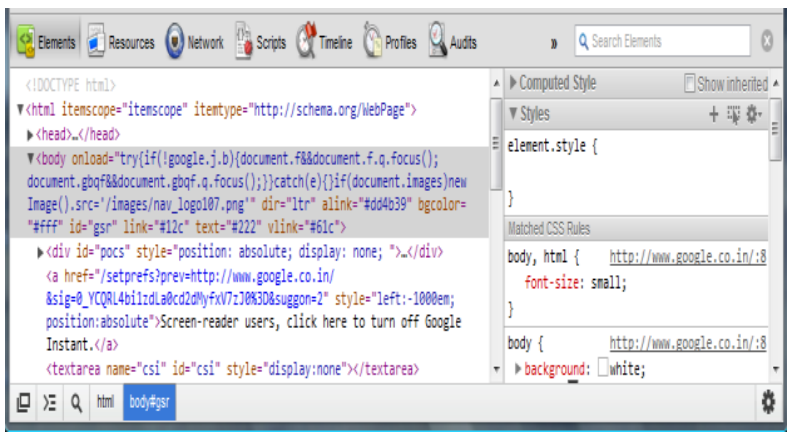

Fig 8: Web developers tool 


\subsection{Memory comparison}

Use this simple trick to compare browsers memory usage. Open a new tab inside Chrome browser and type in the address bar about:memory (or) chrome://memory-redirct. Chrome can track the memory used by itself, but also other browsers currently open and running on your computer.

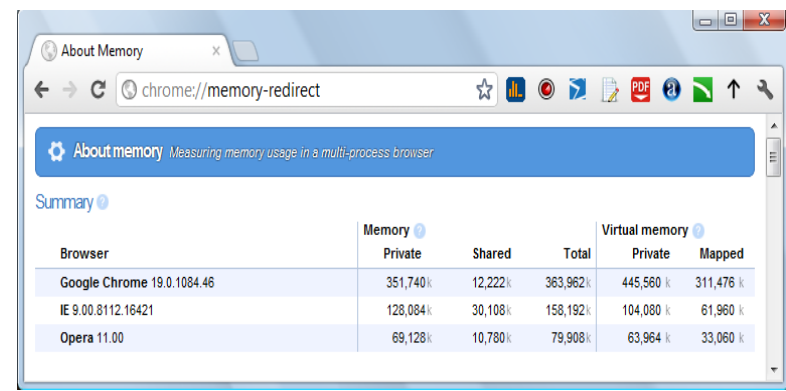

Fig 9: Compare browser memory tool

\subsection{Chrome commands}

Back in 2008, Damien wrote an article going through various Firefox about page tricks. Well, Chrome has similar functions behind the scenes, whereby accessing various about pages lets you turn on and off features that you might not otherwise have access to. They can also provide a window into information that might be useful in debugging a problem you might be having, or useful for some other purpose[12].To view all the available chrome commands, type (chrome://about) in your chrome browser URL as shown below.

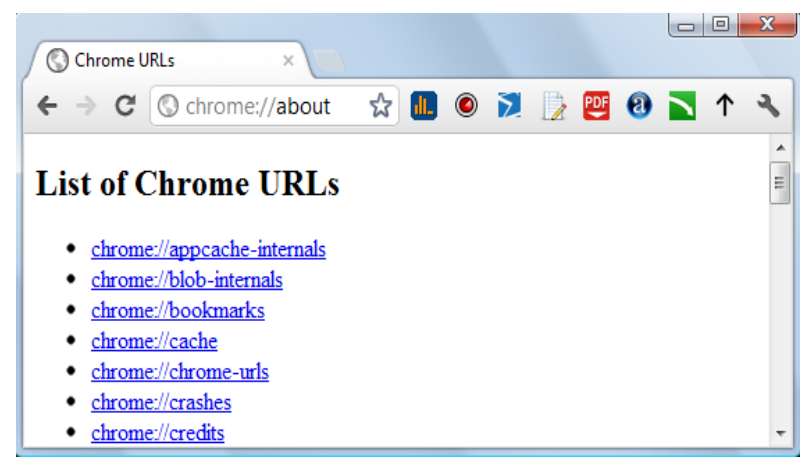

Fig 10: Chrome URL commands

\section{CONCLUSION}

Browsers have become complicated, multi content, multi purpose applications in recent times. Google Chrome has done well in the past few years since it's emerged. The high speed it brings to the internet users, the simply designed but not simple operating selections, has all been astonishing. Google Chrome's has also done a pretty well job on the defense of internet virus. Chrome will no doubt receive most of the attention, if for no other reason than it's from Google and, thus, newer and cooler.

The important thing to remember is that this does not mean that, Chrome is not vulnerable. Vulnerabilities will always be a constant with some technologies having more or less, but your ability to understand your vulnerabilities and manage them will always be the winning formula.

\section{REFERENCES}

[1] The chromium projects, developers, design documents. http://dev.chromium.org/developers/design-documents

[2] Liz Stinson. Browser architecture Chrome, 2009. http://lizstinson.blogspot.com/2009/06/browserarchitecture-chrome.html

[3] Why use google chrome, chrome. https://www.google.com/chrome/intl/en/more/index.html

[4] The Chromium Blog, 2012. news and developments from the open source browser project. http://blog.chromium.org

[5] Google Chrome browser project. 2008, google blogoscoped. http://blogoscoped.com/archive/2008-0901-n47.html.

[6] Dinu. Chrome latest updates, Chrome story http://www.chromestory.com.

[7] Jithin. Get a closer view of google chrome browser features, 2012,sparkyhub. http://www.sparkyhub.com/get-a-closer-view-of-googlechrome-browser-features/.

[8] Making the web faster. Google chrome browser. a http://google-chrome-browser.com/tags/googlechrome?page $=5$

[9] Google chrome and privacy. 2012, static google user content.

http://static.googleusercontent.com/external_content/untr usted_dlcp/www.google.com/en//intl/en/landing/chrome/ google-chrome-privacy-whitepaper.pdf.

[10] The rlz project empowers promotion ROI analysis. google project hosting. http://code.google.com/p/rlz/

[11] Daniel. Google operating system. 2008, google system blogspot.

http://googlesystem.blogspot.com/2008/09/googlechromes-about-pages.html

[12] JJ. Useful chrome about pages. 2011, make tech easier. http://maketecheasier.com/7-useful-chrome-about-pagesyou-didnt-know-exist/2011/09/17

[13] Google chrome. 2012, Wikipedia. http://en.wikipedia.org/wiki/Google_Chrome.

[14] BSD licences, 2012, Wikipedia. http://en.wikipedia.org/wiki/BSD_license.

[15] Chromium browser Vs Google chrome. 2011, code google.

http://code.google.com/p/chromium/wiki/ChromiumBro wserVsGoogleChrome.

[16] Code reuse in google chrome browser. 2008, catonmat. http://www.catonmat.net/blog/code-reuse-in-googlechrome-browser/.

[17] Shane McGlaun. German government recommends chrome browser. 2012, slashgear. http://www.slashgear.com/german-governmentrecommends-chrome-browser-06212198/.

[18] Yiqin Zhu. Research paper in google chrome. 2011, sitesgoogle.

https://sites.google.com/site/zyq0907/research-paper-ingoogle-chrome. 
[19] Joshua Drake, Paul Mehta, Charlie Miller, Shawn Moyer, Ryan Smith, Chris Valasek. 2011, accuvant Labs, Browser security comparison a quantitative approach.http://www.accuvant.com/sites/default/files/im ages/webbrowserresearch_v1_0.pdf.

[20] Vishal Kumar Gaurav. Google chrome Vs Internet Explorer. 2010 ,

scribd http://www.scribd.com/vgaurav_4/d/49403748-GoogleChrome-vs-Internet-Explorer.

\section{AUTHOR PROFILE}

A.Sankara Narayanan is presently working as a Technical Support in Department of Information Technology at Salalah College of Technology, Salalah, Sultanate of Oman. He has 9 years of Networking/System experience and 4 years of Information Security experience. $\mathrm{He}$ has published 8 international journals. His research interests include ethical hacking, computer forensics, malware and information Security. 